\title{
Transient Two-Dimensional Analysis of Side Load in Liquid Rocket Engine Nozzles
}

\author{
Ten-See Wang ${ }^{*}$ \\ NASA Marshall Space Flight Center,Huntsville,Alabama,35812
}

\begin{abstract}
Two-dimensional planar and axisymmetric numerical investigations on the nozzle startup side load physics were performed. The objective of this study is to develop a computational methodology to identify nozzle side load physics using simplified twodimensional geometries, in order to come up with a computational strategy to eventually predict the three-dimensional side loads. The computational methodology is based on a multidimensional, finite-volume, viscous, chemically reacting, unstructured-grid, and pressure-based computational fluid dynamics formulation, and a transient inlet condition based on an engine system modeling. The side load physics captured in the low aspect-ratio, two-dimensional planar nozzle include the Coanda effect, afterburning wave, and the associated lip free-shock oscillation. Results of parametric studies indicate that equivalence ratio, combustion and ramp rate affect the side load physics. The side load physics inferred in the high aspect-ratio, axisymmetric nozzle study include the afterburning wave; transition from free-shock to restricted-shock separation, reverting back to free-shock separation, and transforming to restricted-shock separation again; and lip restricted-shock oscillation. The Mach disk loci and wall pressure history studies reconfirm that combustion and the associated thermodynamic properties affect the formation and duration of the asymmetric flow.
\end{abstract}

\section{Introduction}

Side loads are potentially detrimental to the integrity and life of almost all launch vehicle nozzles. For example, side load problems have been found for $\mathrm{J} 2$ engine $^{1}$, Space Shuttle Main Engine (SSME) Block I, and recently, the Fastrac Engine nozzles. Unfortunately, the current level of understanding of the cause of nozzle side loads is limited and does not allow the accurate prediction of side load severity for new nozzle designs. The lack of a detailed prediction capability results in reduced life and increased weight for reusable nozzle systems. Simple models exist to predict side load magnitudes but are overly conservative and not fully validated. A clear understanding of the mechanisms and factors that contribute to side loads during engine startup, shutdown, and steady-state operations must be attained. Subsequently, a predictive tool must be developed to aid the development of future reusable engines.

In 1992, Wang ${ }^{2}$ reported the first attempt in using computational fluid dynamics (CFD) to study separated flows inside an axisymmetric SSME nozzle. Chen, et al. ${ }^{3}$ reported a similar effort on an axisymmetric cold flow J2S nozzle in 1994, using impulsive starts and cut-offs. Although both captured the nozzle hysteresis phenomenon that is one of the basic characteristics of liquid rocket engine nozzles, strictly speaking, the computed axisymmetric flow features such as Mach disk, separation shock, Mach stem, vortex core, contact surface, slip stream, and shocklets are irrelevant to side load physics due to the axisymmetric constraints.

Under that premise, a cold flow wind tunnel test was conducted at Marshall Space Flight Center's transonic wind tunnel facility in early 1990s, using a two-dimensional (2-D) SSME nozzle shell contour model. Schlieren pictures, static and dynamic fluctuating pressure measurements were made for analysis. Unfortunately the start-up and shutdown sequences were not recorded and the test duration was too long to make it suitable for numerical simulations. The Schlieren pictures did shed some light in the core flow and Mach disk flow physics. For example, it was shown that the core flow adhered to one-side of the wall before the Mach disk flow developed.

As the computational technology advances, transient 2-D and three-dimensional (3-D) CFD analyses have become affordable. Yonezawa, et al. ${ }^{4}$ made the first 2-D nozzle side load prediction in 2002 for the Japanese LE-7A engine. The same group then proceeded to simulate the 3-D side load for the LE-7 and CTP50-R5-L nozzles. ${ }^{5}$ They

\footnotetext{
- Staff Consultant, Applied Fluid Dynamics Analysis Group, TD64, Senior Member AIAA.
} 
showed that the 2-D simulations are useful in investigating the characteristics of the side load, leading to the subsequent 3-D rocket nozzle flow simulations.

The works of Yonezawa, et al. ${ }^{4.5}$ however, ignored the effect of combustion; in addition, a linear ramping was assumed. Since the inlet flow ramping determines the filling rate and the combustion provides the chemical energy for the propulsive flow, these two parameters are considered important to the side load physics and are emphasized in this study. This is accomplished by performing a series of 2-D planar and axisymmetric SSME nozzle start-up analyses, with an enhanced unstructured-grid CFD methodology and a simulated start-up sequence obtained from an engine system calculation. To study the effect of combustion, frozen and finite-rate chemistry analyses were performed. To investigate the effect of ramping, side load physics from ramp times of $5 \mathrm{~s}, 3 \mathrm{~s}$, 1s, and impulsive start were computed. The final results are used to develop a computational strategy for the eventual transient 3-D nozzle investigations.

\section{Computational Fluid Dynamics}

\section{Computational Methodology}

The CFD methodology is based on a multi-dimensional, finite-volume, viscous, chemically reacting, unstructured grid, and pressure-based formulation. Time-varying transport equations of continuity, momentum, global energy (total enthalpy), turbulence, and species continuity were employed. A predictor and corrector solution algorithm was employed to provide coupling of the governing equations. A second-order central-difference scheme was employed to discretize the diffusion fluxes and source terms of the governing equations. For the convective terms, a second-order total variation diminishing difference scheme was used. To enhance the temporal accuracy, a second-order backward difference scheme was employed to discretize the temporal terms. Details of the numerical algorithm can be found in Ref's 6-9.

An extended $k-\varepsilon$ turbulence model ${ }^{10}$ was used to describe the turbulence. A modified wall function approach was employed to provide wall boundary layer solutions that are less sensitive to the near-wall grid spacing. Consequently, the model has combined the advantages of both the integrated-to-the-wall approach and the conventional law-of-the-wall approach by incorporating a complete velocity profile and a universal temperature profile $^{11}$. A 7-species, 9-reaction detailed mechanism ${ }^{11}$ was used to describe the finite-rate, hydrogen/oxygen $\left(\mathrm{H}_{2} / \mathrm{O}_{2}\right)$ afterburning chemical kinetics. The seven species are $\mathrm{H}_{2}, \mathrm{O}_{2}, \mathrm{H}_{2} \mathrm{O}, \mathrm{O}, \mathrm{H}, \mathrm{OH}$, and $\mathrm{N}_{2}$.

\section{Simulated Start-up Sequence}

The thruster inlet properties were obtained from an engine system calculation, simulating the valve sequence. Figure 1 shows some of the inlet flow properties: the time-varying inlet pressure, temperature, and equivalence ratio profiles. And it is hereby referred as the nominal $5 \mathrm{~s}$ start-up sequence, in order to differentiate with the shortened sequence in the ramp rate study. It can be seen that there are two significant pressure rise events. The first one occurs at $1.5 \mathrm{~s}$ due to oxygen prime, while the second one occurs at about $2.4 \mathrm{~s}$, caused by the step opening of the oxygen valve in the pre-burners. It can be seen that the thruster environment is fuel rich throughout the start-up transient, setting up the potential for afterburning. The high equivalence ratio in the first $0.2 \mathrm{~s}$ is not significant since the flow rate is negligible.

\section{Computational Grid Generation}

Parametric studies conducted in Ref. 9 show that a structured-cell dominated axisymmetric hybrid grid ax6 performed better than an unstructured-cell dominated grid both in accuracy and efficiency, on flow physics and prediction of nozzle design parameters such as axial force and heat fluxes. Assuming that a grid suitable for axial force calculation is sufficient for side force calculation, grid ax6 was used in this study for axisymmetric calculations and its layout is shown in Fig. 2. It can be seen that structured (quadrilateral) cells are used in the thruster and plume region, while unstructured (triangular) cells are used in the outer (freestream) region. For 2-D planar nozzle studies, grid $2 \mathrm{~d} 6$ was constructed by mirroring grid ax6 for 180 degrees (not shown). These computational grids were generated using the software package GRIDGEN. ${ }^{12}$ Table 1 shows the total number of points and cells in these two grids.

Table 1. Number of points and cells of the grids.

\begin{tabular}{|l|r|r|r|r|}
\hline grid & \# points & \# cells & \# quadrilateral cells & \# triangular cells \\
\hline ax6 & 17,391 & 17,710 & 15,300 & 2,410 \\
\hline 2d6 & 34,782 & 35,420 & 30,600 & 4,820 \\
\hline
\end{tabular}




\section{Boundary Conditions and Run Matrix}

Fixed total condition was used for the outer boundary and a total pressure of 1 atm was used to simulate the nozzle hot-firing at sea level. No-slip condition was specified for the solid walls. The inlet flow properties obtained from the engine system simulation include the time varying total pressure and temperature, and propellant composition. The time varying propellant composition was preprocessed with the Chemical Equilibrium Calculation program $^{13}$, assuming the propellants were ignited to reach equilibrium composition immediately at the injector faceplate. The fuel rich environment indicates the time-varying inlet composition contains essentially steam and excess hydrogen.

The run matrix is shown in Table 2 . These cases were designed to identify side load physics under simplified geometries such as the 2-D planar nozzles. The low-aspect ratio characteristics of the 2-D planar nozzle, however, limit the range of physics to be captured. Axisymmetric studies were therefore followed to complete the picture in the high aspect-ratio nozzle regime. Although axisymmetric model precludes the asymmetric flow formation, the axial shock oscillation caused wall pressure oscillation can still be used to infer side load physics because it leads to side forces if the symmetric constraint is removed. As shown in Table 2, the effect of thermochemistry was investigated for both the 2-D planar and axisymmetric nozzles, whereas the effect of ramp rate was studied under the 2-D planar nozzles only. Adiabatic wall was used for all cases. For finite-rate chemistry cases, the chemical reactions are turned on at the start command, indicating any chemical reaction computed is afterburning of the excess $\mathrm{H}_{2}$.

Table 2. Run matrix

\begin{tabular}{|c|c|c|c|}
\hline geometry & chemistry & propellant & ramp time \\
\hline 2d planar & finite-rate & $\mathrm{H}_{2} / \mathrm{O}_{2}$ & $5 \mathrm{~s}$ \\
\hline 2d planar & frozen & $\mathrm{H}_{2} / \mathrm{O}_{2}$ & $5 \mathrm{~s}$ \\
\hline 2d planar & finite-rate & $\mathrm{H}_{2} / \mathrm{O}_{2}$ & $3 \mathrm{~s}$ \\
\hline 2d planar & finite-rate & $\mathrm{H}_{2} / \mathrm{O}_{2}$ & $1 \mathrm{~s}$ \\
\hline 2d planar & finite-rate & $\mathrm{H}_{2} / \mathrm{O}_{2}$ & impulsive \\
\hline axisymmetric & finite-rate & $\mathrm{H}_{2} / \mathrm{O}_{2}$ & $5 \mathrm{~s}$ \\
\hline axisymmetric & frozen & $\mathrm{H}_{2} / \mathrm{O}_{2}$ & $5 \mathrm{~s}$ \\
\hline axisymmetric & frozen & air & $5 \mathrm{~s}$ \\
\hline
\end{tabular}

\section{Results and Discussion}

The computations were performed on a cluster machine using 2 8 processors for each case. For the $5 \mathrm{~s}, 3 \mathrm{~s}$, and $1 \mathrm{~s}$ start-up sequences, global time steps of 10,5 , and $2.5 \mu \mathrm{s}$ were used in the beginning, respectively, and reduced to $1 \mu \mathrm{s}$ after the first pressure rise. For the impulsive start, $1 \mu \mathrm{s}$ was used throughout the course of the computation.

\section{Two-dimensional Thruster}

The first case in Table 2 is the baseline case for the 2-D planar nozzle. As indicated in Fig. 1, the pressure rises rather slowly, after the start command. Yet the chamber gradually fills up and a detached jet emerges from the throat. As the jet Mach number increases with the rising pressure, the jet shear layer becomes unstable and entrains the surrounding air. Due to the viscous flow process, the fluid that is entrained is accelerated slightly more on oneside than the other side, or the pressure on the slightly accelerated side is lower than the other side, causing the core jet to curve towards the wall (the slightly accelerated side), as shown in the Mach number contours at $0.4 \mathrm{~s}$ in Fig. 3. That further reduces the pressure there, and eventually the jet adheres itself to the wall, enclosing a separation bubble at $0.6 \mathrm{~s}$ (not shown). The separation bubble changes its size incessantly and the attached region can be as long as a-quarter of the nozzle length from the lip. This wall-sticking phenomenon is known as the Coanda effect, ${ }^{14,15}$ after Henri Coanda for his study of jets that are frequently observed to adhere to and flow around nearby solid boundaries. This phenomenon was observed in the afore-mentioned cold flow wind tunnel test.

The flow chokes around $1.025 \mathrm{~s}$ where a Mach disk flow develops. The Mach disk flow contours at 1.35, 1.4 and $1.5 \mathrm{~s}$ are shown in Fig. 3. At $1.35 \mathrm{~s}$, the expanding-contracting supersonic jet still adheres itself to the lip. At 1.4 $\mathrm{s}$, the Mach disk is fully developed. The upper jet adheres to the wall, enclosing a recirculation region, forming a so- 
called "restricted-shock separation (RSS)"; while the lower jet flows freely, forming a so-called "free-shock separation (FSS)". At $1.5 \mathrm{~s}$, both supersonic jets are off the wall, exhibiting a classic free-shock separation flow pattern.

The Mach disk propagates out of the nozzle at $1.512 \mathrm{~s}$, as shown in Fig. 4. At this time a strong afterburning occurs, and a combustion wave ensues. That wave burns back the fuel, resulting in an asymmetric Mach disk flow retreating back into the nozzle, as shown in a series of plots at $1.515,1.516$, and $1.520 \mathrm{~s}$. This is the effect of combustion and is further demonstrated in the temperature, pressure, and $\mathrm{H}_{2}$ mass fraction contours shown from Fig. 5 to Fig. 7. The Mach disk then moves out of the nozzle for good, as shown in Fig. 8. The occurrence of the afterburning wave is closely associated with the inlet equivalence ratio history.

Figure 9 shows the computed 2-D planar nozzle side loads. As shown in the top plot of Fig. 9 (the finite-rate chemistry case), the Coanda effect does not create significant side forces on the core jet flow $(0.4 \sim 1 \mathrm{~s})$, but it does on the Mach disk flow ( $1 \sim 1.5 \mathrm{~s}$ ); where the Mach disk flows adhere mostly to the upper wall, thereby producing largely negative side forces. The largest side force is generated after $1.512 \mathrm{~s}$, due to the afterburning wave, although for a very short time. The side force disappears after the Mach disk moves out of the nozzle for good. In the frozen chemistry case, as shown in the bottom plot of Fig. 9, the side forces are mostly positive between 1 to $1.5 \mathrm{~s}$, indicating the Mach disk flow adheres to the lower wall. In addition, the side force is negligible after $1.512 \mathrm{~s}$ because the nozzle remains fully filled. This says without combustion, not only the afterburning induced side load physics is lacking, the captured side force (due to Mach disk flow) is lower in magnitude and acted on the opposite wall. Figure 10 shows the computed Mach disk loci for the two cases. It can be seen that the Mach disk locus of the frozen chemistry never retreats back into the nozzle. On the other hand, that of the finite-rate chemistry case dips deep into the nozzle, showing the effect of burning back the excess fuel.

The effect of ramp rate on side loads is shown in Fig. 11. This is accomplished by running three more cases: ramp times of 3 and $1 \mathrm{~s}$, and an impulsive start case. Comparing to the baseline case in Fig. 9, the ramp rate is increased 1.67 times by shortening the $5 \mathrm{~s}$ ramp time to that of $3 \mathrm{~s}$; hence the first pressure rise occurs at $0.9 \mathrm{~s}$ (instead of the nominal $1.5 \mathrm{~s}$ ). Similarly, the ramp rate is increased five times by shortening the original $5 \mathrm{~s}$ ramp time to that of $1 \mathrm{~s}$, and the first pressure rise event happens at $0.3 \mathrm{~s}$. The impulsive start represents an infinite increase in ramp rate, although the steady-state operation conditions have to be used which actually keep the amount of excess fuel to a minimum. The results in Fig. 11 shows that the side forces due to Coanda effect diminish as the ramp time drops from $5 \mathrm{~s}$ (Fig. 9), to 3, 1, and $0 \mathrm{~s}$ (impulsive start), indicating the adhering ability disappears as the speed of Mach disk flow increases. On the other hand, the side-load caused by the afterburning wave increases as the ramp time decreases from $5 \mathrm{~s}$, to $3 \mathrm{~s}$, and $1 \mathrm{~s}$. In fact, not only the amplitude of the side load increases, the duration of that side load acting on the nozzle increases as well. This is understandable since the strength of the afterburning wave increases, as the pressure ramp rate increases. For the impulsive start case however, neither is there enough fuel for afterburning, nor is there enough residence time for any fuel to burn, hence there is no apparent combustion wave induced side load; note that the computed (impulsive start induced) blast wave leaves the nozzle at $1.75 \mathrm{~ms}$ and the Mach disk leaves the nozzle at $4.25 \mathrm{~ms}$. The result of Fig. 11 demonstrates that ramp rate is another important side load driving force, probably more so than the pressure ratio.

\section{Axisymmetric thruster}

Since 2-D planar nozzles are low aspect-ratio nozzles, axisymmetric investigations followed to infer the side load physics occurring in high aspect-ratio nozzles. The sixth case in Table 2 is the baseline case for the axisymmetric SSME nozzle. The Coanda effect captured in the 2-D planar nozzle studies is precluded due to symmetric boundary conditions. Figures $12-13$ show the computed Mach number, temperature, pressure, and $\mathrm{H}_{2}$ mass fraction contours for the baseline case. It can be seen that afterburning occurs at $1.504 \mathrm{~s}$ (temperature contours), resulting in a combustion wave at $1.505 \mathrm{~s}$ (pressure contours). This combustion wave bounces around in the nozzle at 1.506 and $1.507 \mathrm{~s}$ (pressure contours), and causes a burn-back of the excess fuel $\left(\mathrm{H}_{2}\right.$ mass fraction contours), and the retreat of the Mach disk.

Soon after the afterburning wave event, the Mach disk flow regains its strength, propagating downstream. Figure 14 shows a unique physics not seen in the low aspect-ratio 2-D planar nozzle at this stage. That is, the expandingcontracting supersonic jet originating from the triple point of the Mach disk adheres to the wall at $1.525 \mathrm{~s}$, enclosing a recirculation region, forming a classic RSS flow pattern. This of course is triggered by the Coanda effect. Then the supersonic jet goes off the wall once more at $1.55 \mathrm{~s}$ and re-adheres itself to the wall again at $1.6 \mathrm{~s}$ and never goes off the wall thereafter, until it leaves the nozzle. This phenomenon of transiting form FSS (1.5 s) to RSS (1.525 $\mathrm{s}$ ), reverting back to FSS (1.55 s), and transforming back to RSS again, increases the side forces.

As the Mach disk approaches the lip, eventually, the first stem (foot) of the two stems (feet) that constitute the RSS steps out of the nozzle at $2.875 \mathrm{~s}$, as shown in Fig. 15. It is expected that this phenomenon would generate the 
largest side force and is named the "lip restricted-shock oscillation". A so-called "tepee" shock pattern would have resulted near the lip if this were a three-dimensional calculation. The separated flow and the Mach disk are completely driven out of the nozzle around $3.4 \mathrm{~s}$. Once the Mach disk retreats back into the nozzle at $3.45 \mathrm{~s}$, it leaves the nozzle forever at $3.7 \mathrm{~s}$.

Figure 16 shows the Mach disk loci of axisymmetric nozzle for three cases: finite-rate chemistry, frozen chemistry, and cool air. It has been shown (Fig. 10) previously in the low aspect-ratio nozzle (2-D planar) that the finite-rate chemistry caused the Mach disk to retreat back into the nozzle, showing a dip that passes the nozzle lip location, while the frozen chemistry cause the disk to retreat only a small distance. In Fig. 16, similar phenomenon happens (see the insert), except it occurs way deep inside the nozzle, because the axisymmetric nozzle has a very high aspect-ratio. Figure 16 also shows that the head (center) of the Mach disk of the frozen flow case does not leave the lip very far. This agrees with the finding in Ref. 9 where the result of the steady-state frozen flow showing a curved disk that curves towards the lip. It was reasoned that the frozen flow composition forces higher specific heat ratios, which create higher Mach numbers, which in turn generate more total pressure loss across the shock, thereby resulting in a curved disk that appears closer to the lip (than that of the finite-rate chemistry case). With that in mind, the cool air case was run because it has a constant and even higher specific ratio (1.4). The result shows that cool air as the working fluid gives qualitatively similar nozzle flow pattern, e.g., the formations of FSS and RSS, although the transition of which occurs later than the hot hydrogen fueled flows. By definition, however, the combustion-driven flow phenomena such as the afterburning can not be captured. In addition, under the same numerical operating conditions (i.e., nozzle contour and pressure ramp sequence), its Mach disk flow just oscillates inside the lip, as indicated in Fig. 16.

Figures 17-18 show the pressure histories of the afore-mentioned three cases at two wall points. The first wall point is located at approximately the middle of the nozzle (Fig. 17), while the second wall point is located just off the nozzle lip (Fig. 18). In general, it can be seen that the pressure histories are quite different for all three cases, when compared at the same location. It should be noted that the timing of the two side load events (the afterburning wave and transition from FSS to RSS) after $1.5 \mathrm{~s}$ are very close (Fig's 12-14) and almost indistinguishable in the pressure history plot. It can therefore be reasonably assumed that the first pressure peak in the finite-rate case is caused by a combination of the afore-mentioned two events, whereas the lower first peak value for the frozen chemistry case comes from the transition from FSS to RSS only. Note that the occurrence of the first peak in the cool air case that is also caused by the shock transition is delayed and the amplitude is even lower. Other than the first peak event, the amplitude of pressure oscillation of the frozen flow case is higher than that of the finite-rate case at all points, but the duration of oscillation becomes longer for the frozen flow case at the last wall point. This is because by $3.5 \mathrm{~s}$, the Mach disk of the finite-rate case has long left the nozzle, while the curved Mach disk of the frozen flow case is lingering around the nozzle lip; And the amplitude of pressure oscillation for the cool air case is lower at the first wall point (Fig. 17), due to less energetic flows, but it grows very high at the second wall point (Fig. 18) and the duration of the oscillation lasts until the end. This is because the Mach disk of the cool air case never leaves the nozzle. The result of the cool air case agrees with the assertion ${ }^{16}$ that cold-gas test results cannot be reliably used for direct extrapolation to full-scale because the gas composition is significantly different from typical full-scale test conditions. In summary, while the results from the computed Mach disk loci and wall pressure histories are explainable by the distribution of thermochemical properties, it should be noted that it is the combustion process that determines the right distribution of the thermochemical properties inside the nozzle, which in turn drives the side load physics occurring in the propulsive nozzles.

\section{Conclusions}

A computational methodology was developed to study the side load physics during the simulation of hot-firing of liquid rocket engines with simplified 2-D geometries, so as to come up with a computational strategy to predict the 3-D side loads. The computational methodology is based on an unstructured grid and pressure-based computational fluid dynamics formulation, and a transient inlet condition based on an engine system simulation. In the low aspect-ratio, 2-D planar nozzle study, side load physics captured include the Coanda effect on both the core jet and Mach disk flows, afterburning wave originated outside of the nozzle, and the associated free-shock oscillation at the lip. In addition, all captured side load physics are profoundly impacted by ramp rate, equivalence ratio, and combustion. In the high aspect-ratio, axisymmetric nozzle study, side load physics inferred include the afterburning wave originated inside of the nozzle; transition from FSS to RSS, reverting back to FSS, and transforming back to RSS again; and restricted-shock oscillation at the lip. In the Mach disk loci and the wall pressure history studies, it is found that chemical reactions produce the right thermochemical properties, which in 
turn impact the side load physics. These results indicate that a proper inlet history and the inclusion of combustion modeling are crucial to the investigation of side load physics during simulation of liquid rocket engine nozzle firings.

\section{Acknowledgments}

This study was partially supported by a MSFC FY03 CDDF effort entitled "Nozzle Side Load Technology" of which Joe Ruf is the principal investigator who conducted experimental and simplified analytical studies. Discussions with Werner Dahm and Joe Ruf, Wallace Welder of Boeing Rocketdyne, and Y.-S. Chen of Engineering Sciences, Inc. are appreciated. David Seymour provided the initial start-up information. Robert Garcia suggested the calculation using air as the working fluid.

\section{References}

${ }^{1}$ Nave, L.H., and Coffey, G.A., "Sea Level Side Loads in High-Area-Ratio Rocket Engines,” AIAA Paper 73-1284, Nov. 1973.

${ }^{2}$ Wang, T.-S., "Numerical Study of the Transient Nozzle Flow Separation of Liquid Rocket Engines," Computational Fluid Dynamics Journal, Vol. 1, No. 3, Oct. 1992, pp. 319-328.

${ }^{3}$ Chen, C.L., Chakravathy, S.R., and Hung, C.M., "Numerical Investigation of Separated Flows," AlAA Journal, Vol. 32, No. 9, Sept. 1994, pp. 1836-1843.

${ }^{4}$ Yonezawa, K., Yokota, K., Watanabe, Y., Tsujimoto, K., and Abe, T., "2-D Numerical Simulation of Side Loads in Rocket Nozzles," Proceedings of $23^{\text {rd }}$ International Symposium on Space Technology and Science, Japan Society for Aeronautical and Space Sciences, 2002, pp. 1146-1151.

${ }^{5}$ Yonezawa, K., Yokota, K., Tsujimoto, K., Sakazume, N., and Watanabe, Y., "Three-Dimensional Unsteady Flow Simulation of Compressed Truncated Perfect nozzles," AIAA Paper 2002-3991, July 2002.

${ }^{6}$ Chen, Y.-S., Liu, J., Zhang, S., and Mallapragada, P., "An Integrated Tool for Launch Vehicle Base-Heating Analysis," Final Report, Engineering Sciences, Inc., Huntsville, AL, December, 2001.

${ }^{7}$ Wang, T.-S., Chen, Y.-S., Liu, J., Myrabo, L.N., and Mead, F.B. Jr., "Advanced Performance Modeling of Experimental Laser Lightcraft," Journal of Propulsion and Power, Vol. 18, No. 6, November-December, 2002, pp. 1129-1138.

${ }^{8}$ Chen. Y.-S., Zhang S., and Liu, J., "Stage Separation Performance Analysis Project," Final Report, Engineering Sciences, Inc., Huntsville, AL, June, 2002.

"Wang, T.-S., "Multidimensional Unstructured-Grid Liquid Rocket Engine Nozzle Performance and Heat Transfer Analysis," AIAA Paper 2004-4016, July 2004.

${ }^{10}$ Chen, Y.-S., and Kim, S.W., "Computation of Turbulent Flows Using an Extended k- $\varepsilon$ Turbulence Closure Model," NASA CR-179204, Oct. 1987.

${ }^{11}$ Wang, T.-S., Droege, A., D'Agostino, M., Lee, Y.-C., and Williams, R., "Base-Bleed Effect on X-33 Aerospike Plume Induced Base-Heating Environment During Power-Pack Out," AIAA Paper 2003-3651, June 2003.

${ }^{12}$ Steinbrenner, J.P., Chawner, J.R., and Fouts, C., "Multiple Block Grid Generation in the interactive Environment," AIAA Paper 90-1602, June 1990.

${ }^{13}$ Svehla, R.A., and McBride, B.J., "FORTRAN IV Computer Program for Calculation of Thermodynamic and Transport Properties of Complex Chemical Systems," NASA TN D-7056, Jan. 1973.

${ }^{14}$ Kumada, M., Mabuchi, I., and Oyakawa, K., "Studies on Heat Transfer to Turbulent Jets with Adjacent Boundaries," Bulletin of the Japan Society of Mechanical Engineers, Vol. 16, No. 100, 1973, pp. 1712-1722.

${ }^{15}$ Bourque, C., and Newman, B.G., "Reattachment of a Two-Dimensional, Incompressible Jet to an Adjacent Flat Plate," Aeronautical Quarterly, Vol. 11, No. 8, 1960, pp. 201-232.

${ }^{16}$ Hageman, G., Alting, J., and Preclik, D., "Scalability for Rocket Nozzle Flows Based on Subscale and Full-Scale Testing," Journal of Propulsion and Power, Vol. 19, No. 3, 2003, pp.321-331. 


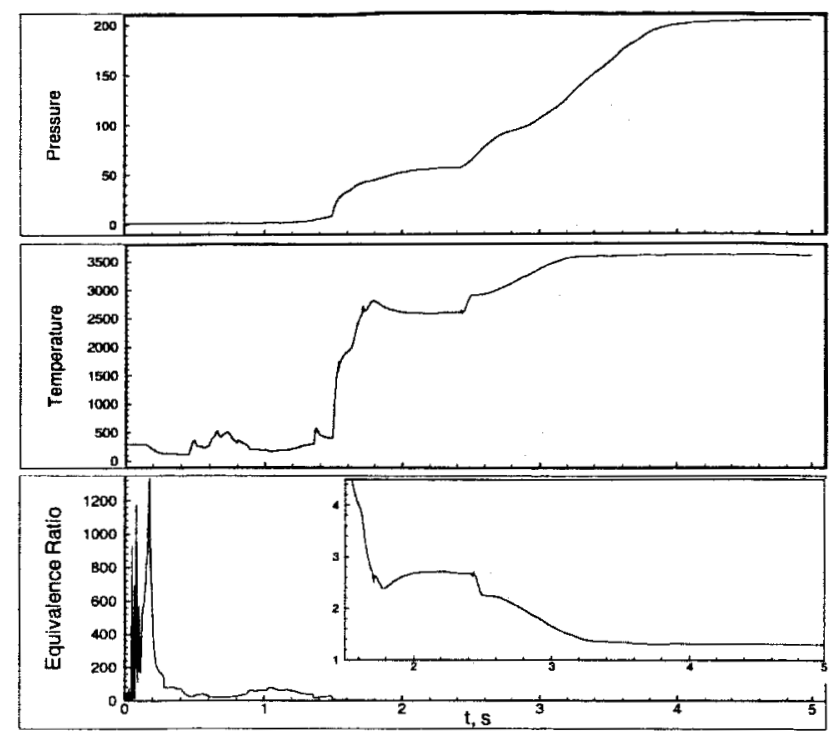

Fig. 1 Simulated thruster inlet properties during the start-up transient.

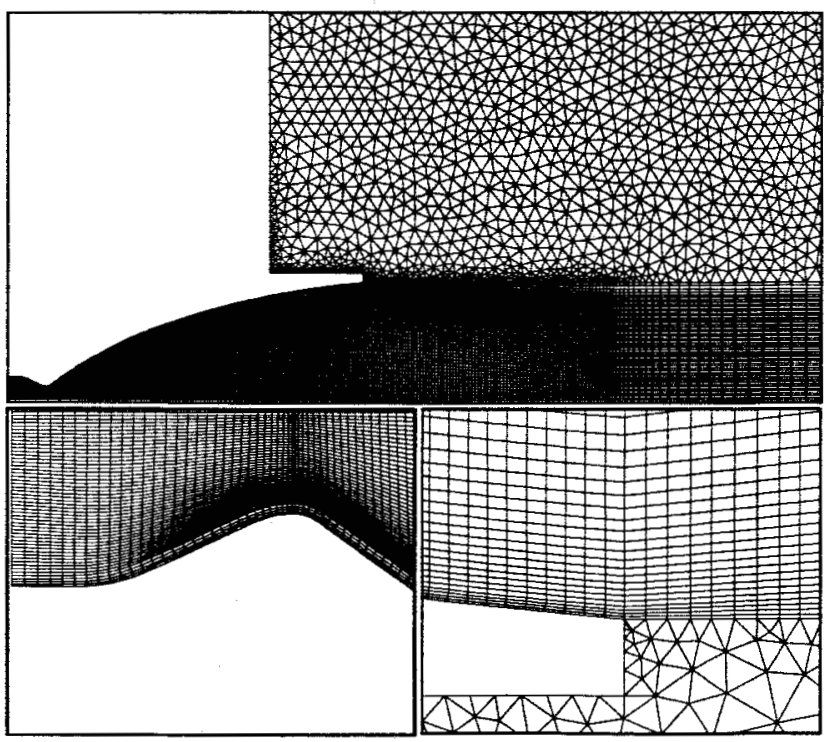

Fig. 2 The layout of hybrid grid ax6. Top: the overall grid. Bottom left: close-up near the throat. Bottom right: close-up near the nozzle lip.

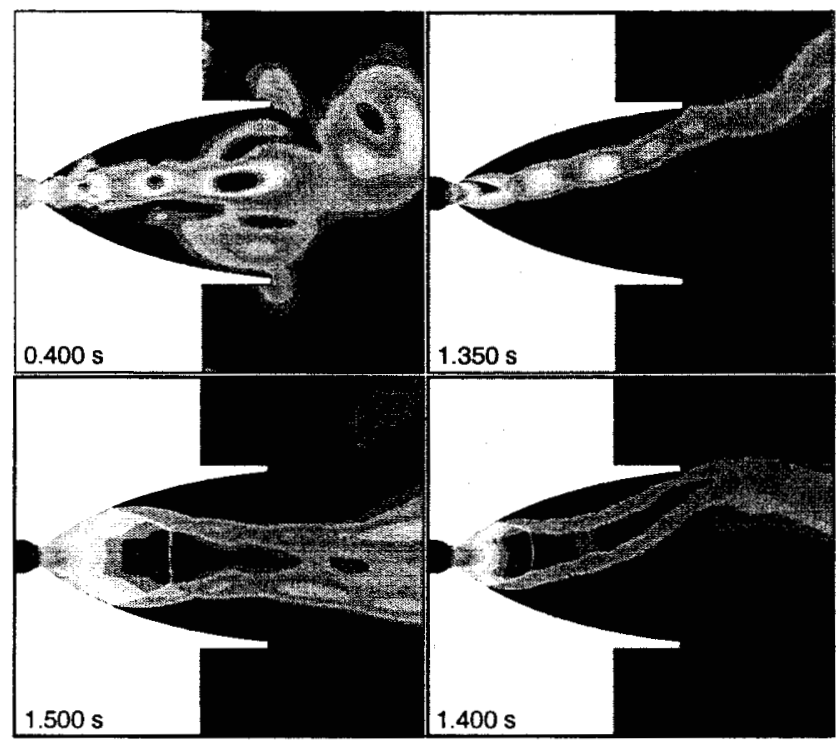

Fig. 3 Computed Mach number contours of baseline 2-D planar nozzle.

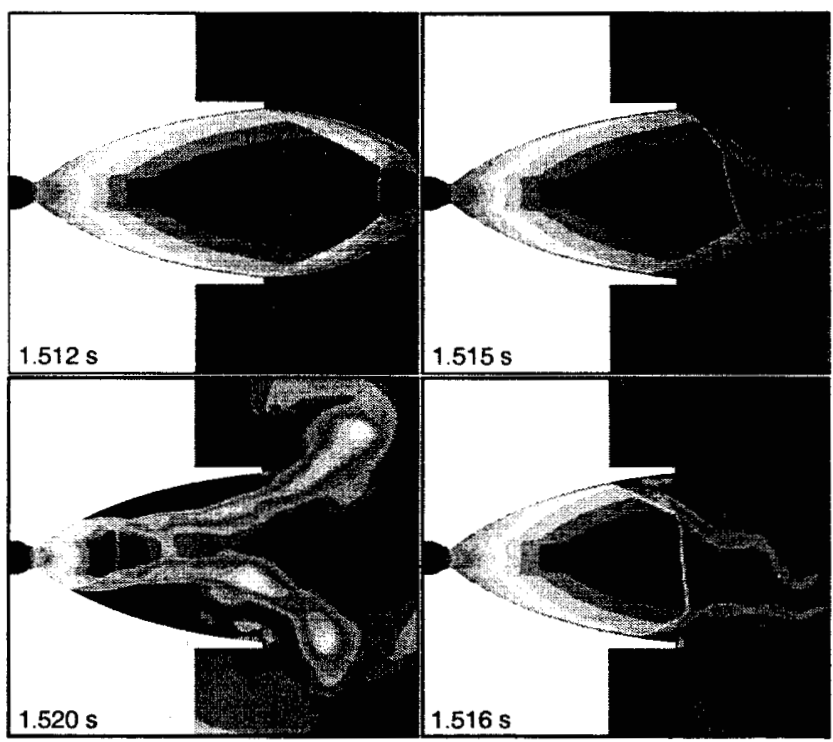

Fig. 4 Computed Mach number contours of baseline 2-D planar nozzle. 


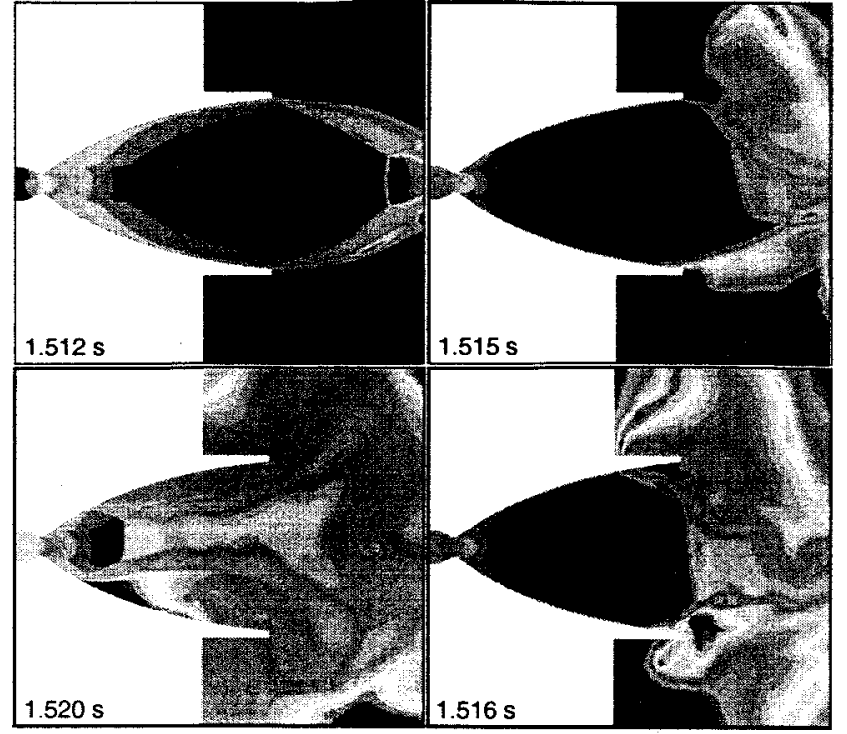

Fig. 5 Computed temperature contours of baseline 2D planar nozzle.

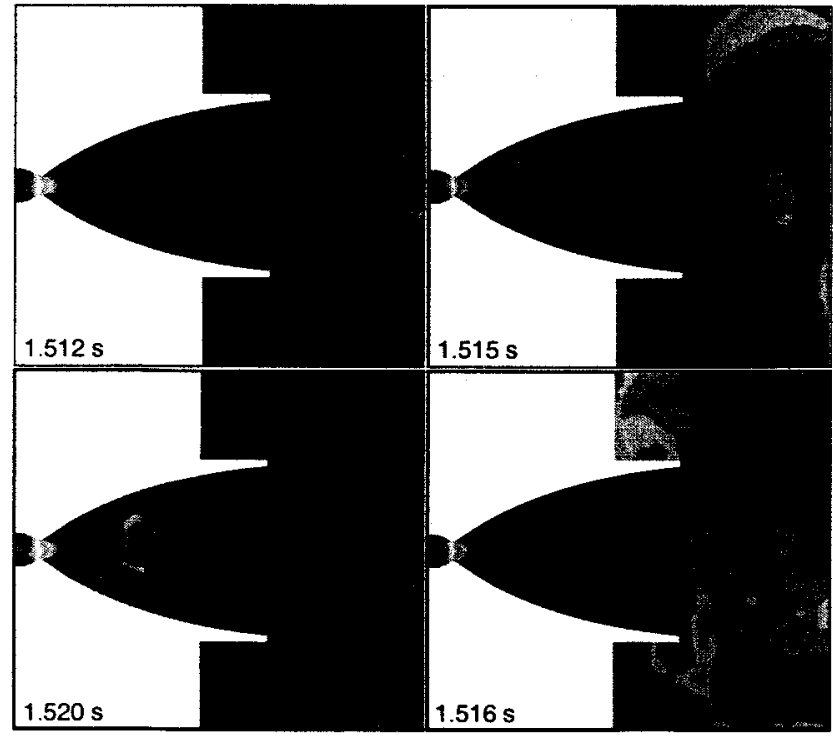

Fig. 6 Computed pressure contours of baseline 2-D planar nozzle.

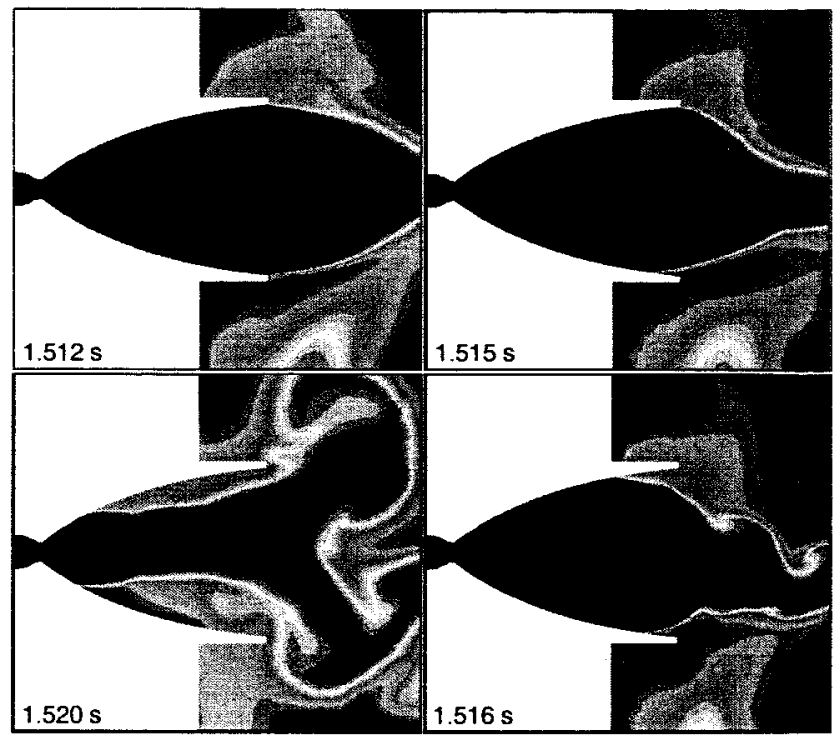

Fig. 7 Computed Hydrogen mass fraction contours of baseline 2-D planar nozzle.

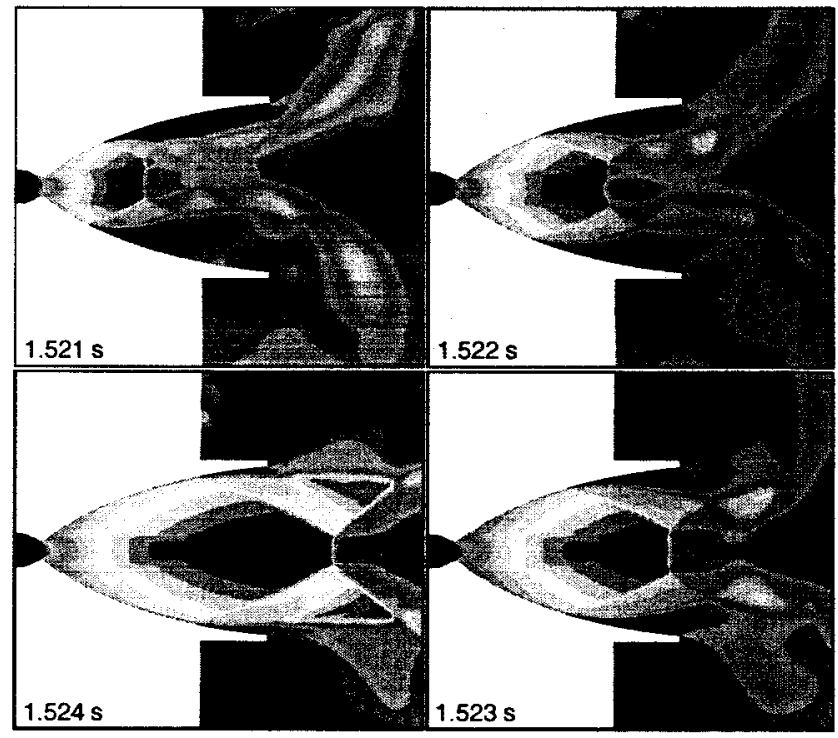

Fig. 8 Computed Mach number contours of baseline 2-D planar nozzle. 


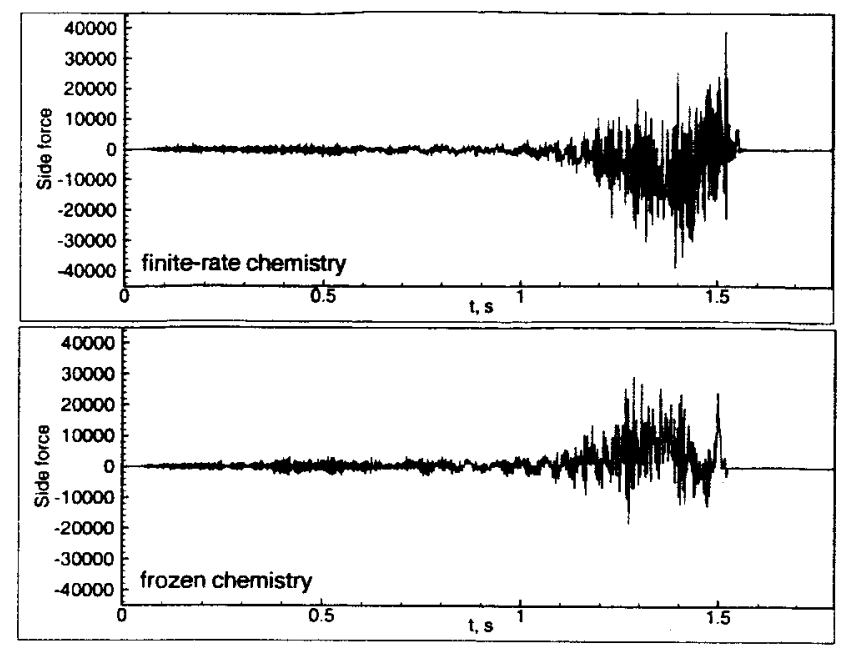

Fig. 9 Effect of chemistry on 2-D planar nozzle side loads.

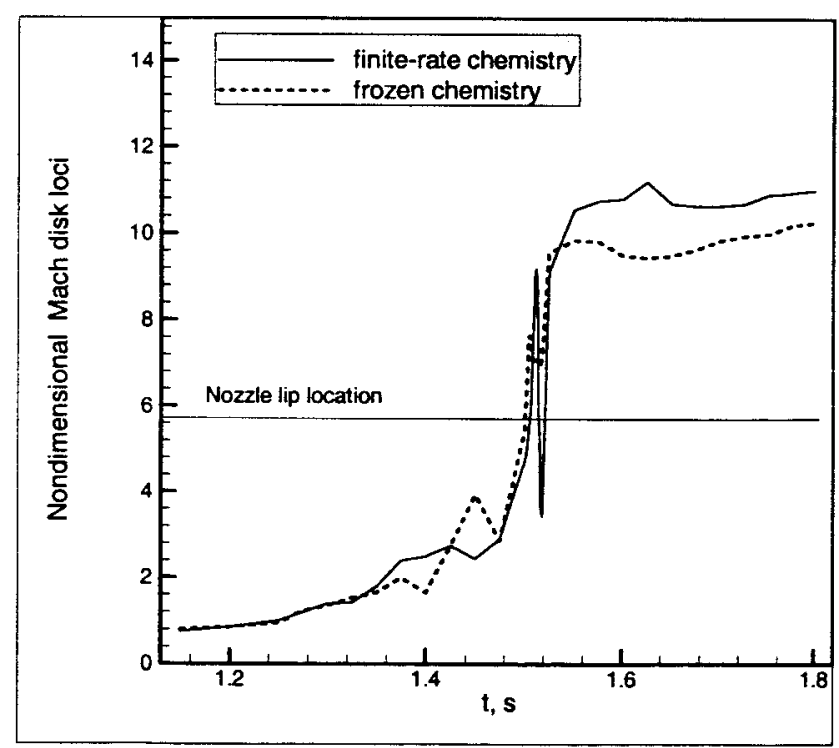

Fig. 10 Computed Mach disk loci for 2-D planar nozzle.

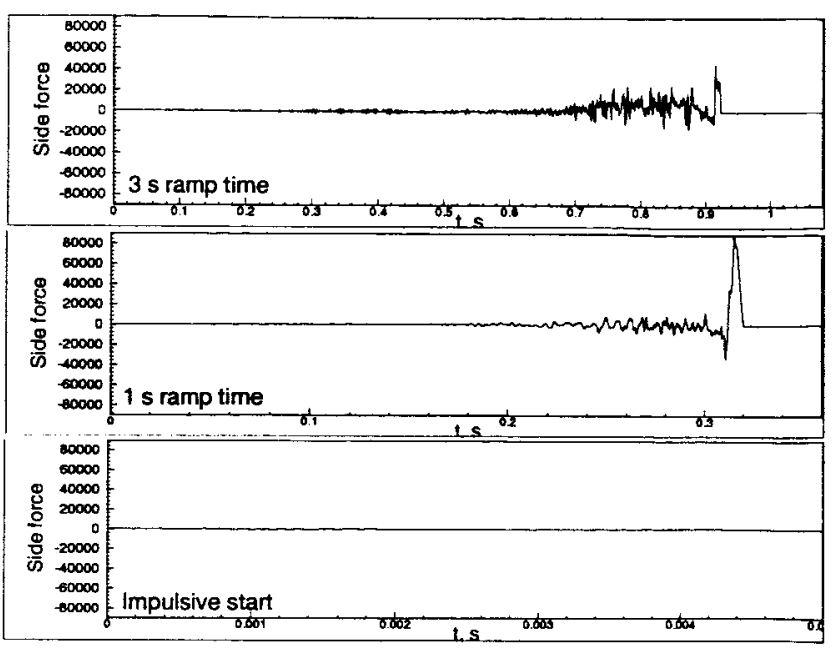

Fig. 11 Effect of ramp rate on 2-D planar nozzle side loads.

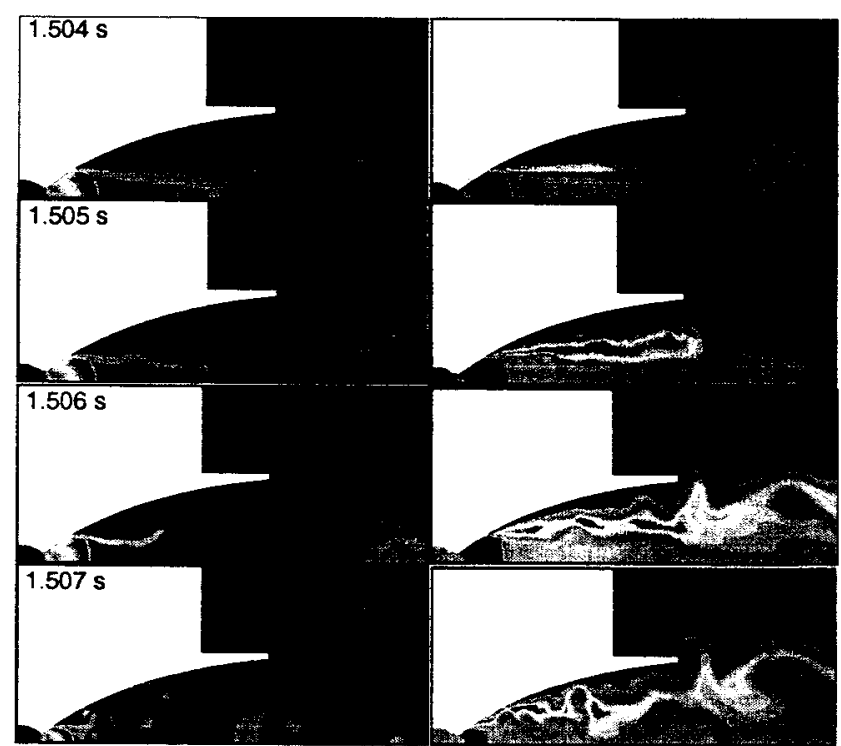

Fig. 12 Computed axisymmetric nozzle Mach number (left column) and temperature (right column) contours. 


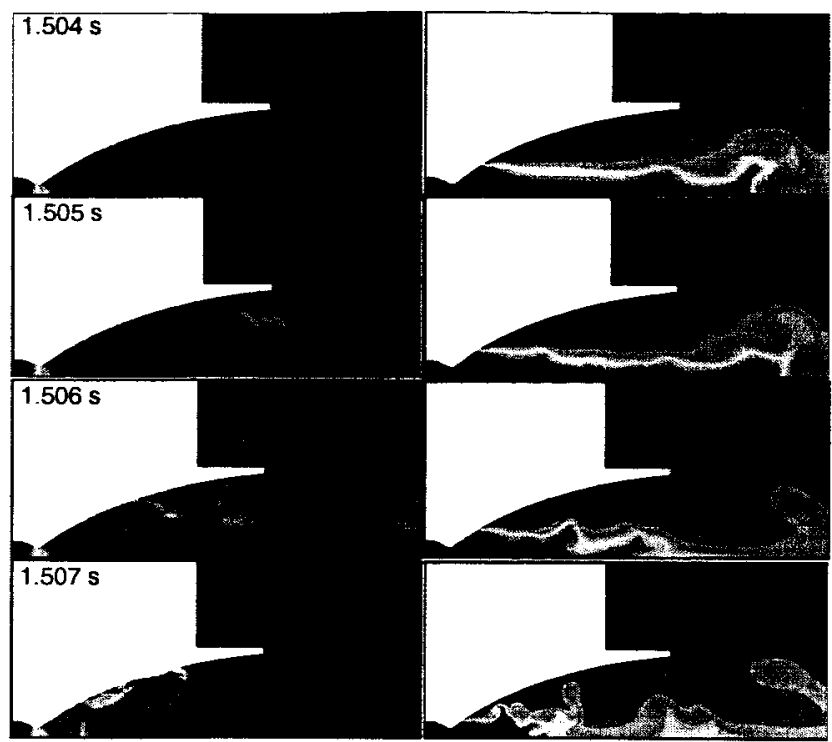

Fig. 13 Computed axisymmetric nozzle pressure (left column) and hydrogen mass fraction (right column) contours.

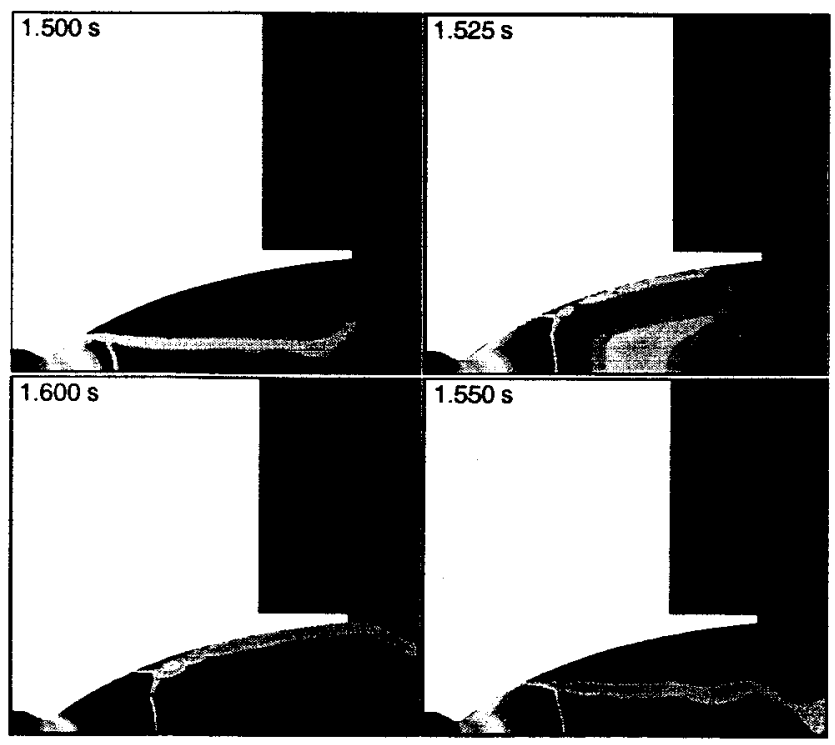

Fig. 14 Computed axisymmetric Mach number contours.

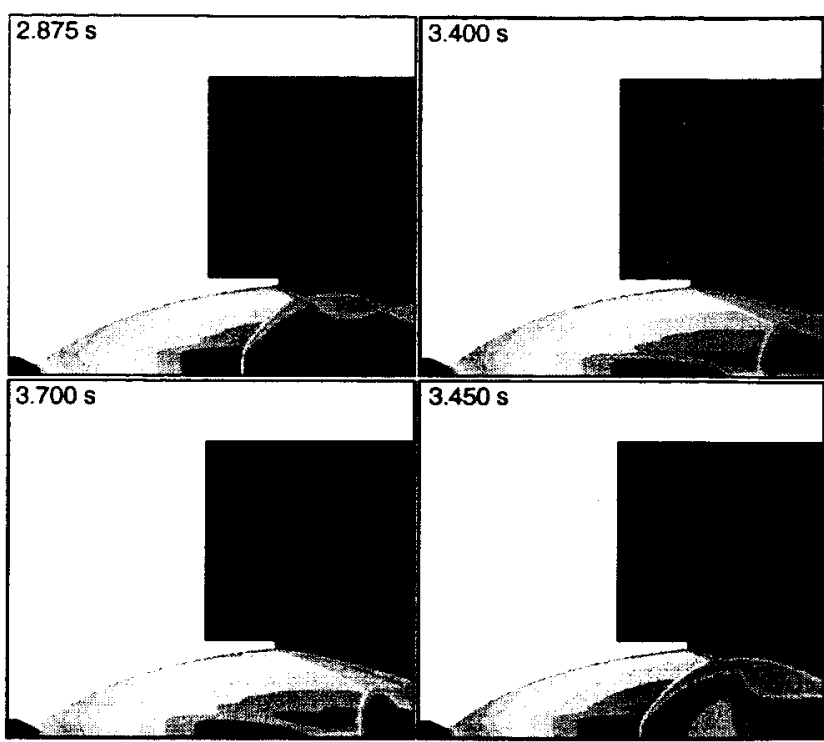

Fig. 15 Computed axisymmetric nozzle Mach number contours.

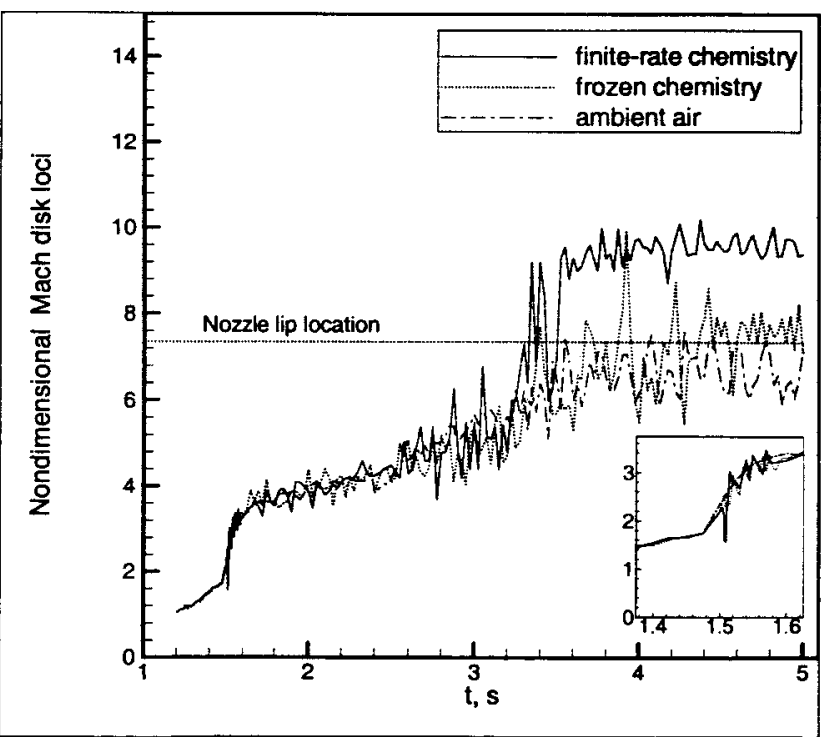

Fig. 16 Computed Mach disk loci for axisymmetric nozzles. Insert: A close-up of the effect of detonation. 


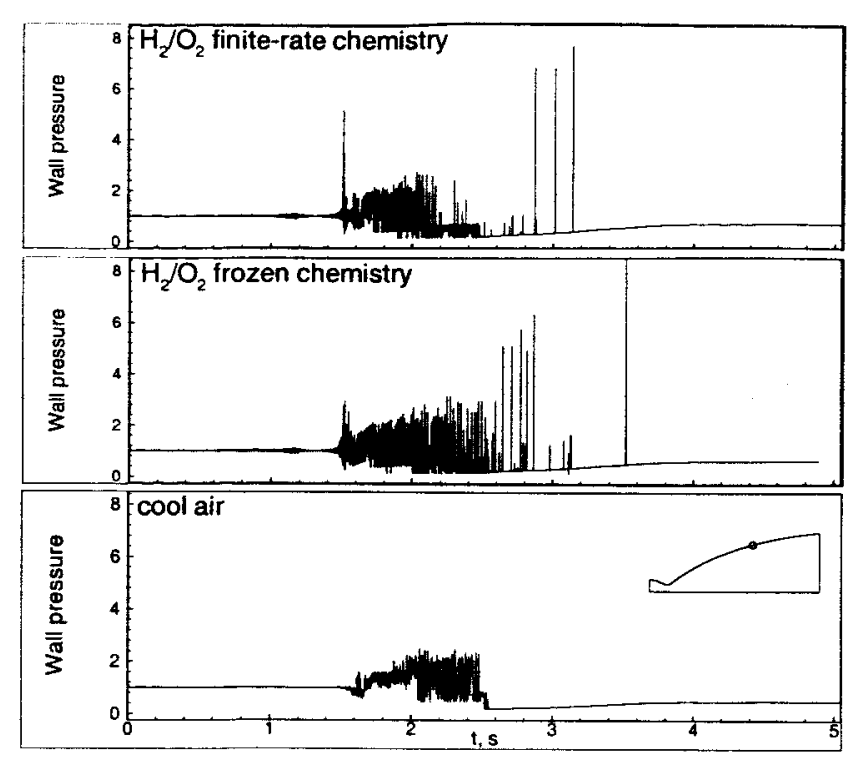

Fig. 17 Computed axisymmetric nozzle wall pressure histories.

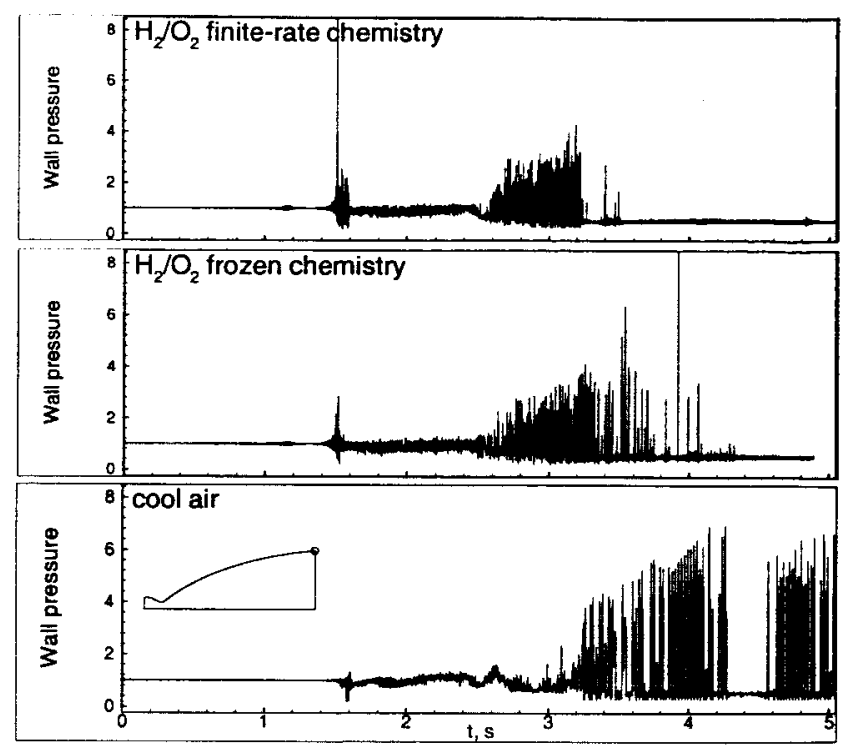

Fig. 18 Computed axisymmetric nozzle wall pressure histories. 